\title{
Temperature and Magnetic Field Enhanced Hall Slope of a Dilute 2D Hole System in the Ballistic Regime
}

\author{
X. P. A. Gao, ${ }^{1, \text { ⿴囗十 }}$ G. S. Boebinger, ${ }^{2}$ A. P. Mills Jr., ${ }^{3}$ and A. P. Ramirez, L. N. Pfeiffer, and K. W. West ${ }^{4}$ \\ ${ }^{1}$ Los Alamos National Laboratory, Los Alamos, NM 87545 \\ ${ }^{2}$ National High Magnetic Field Laboratory, Florida State University, Tallahassee, FL 32312 \\ ${ }^{3}$ Physics Department, University of California, Riverside, CA 92507 \\ ${ }^{4}$ Bell Laboratories, Lucent Technologies, Murray Hill, NJ 07974
}

(Dated: August 26, 2018)

\begin{abstract}
We report the temperature $(T)$ and perpendicular magnetic field $(B)$ dependence of the Hall resistivity $\rho_{x y}(B)$ of dilute metallic two-dimensional(2D) holes in GaAs over a broad range of temperature $(0.02-1.25 \mathrm{~K})$. The low $B$ Hall coefficient, $R_{H}$, is found to be enhanced when $T$ decreases. Strong magnetic fields further enhance the slope of $\rho_{x y}(B)$ at all temperatures studied. Coulomb interaction corrections of a Fermi liquid(FL) in the ballistic regime can not explain the enhancement of $\rho_{x y}$ which occurs in the same regime as the anomalous metallic longitudinal conductivity. In particular, although the metallic conductivity in $2 \mathrm{D}$ systems has been attributed to electron interactions in a FL, these same interactions should reduce, not enhance the slope of $\rho_{x y}(B)$ as $T$ decreases and/or $B$ increases.

PACS numbers: 71.30.+h, 73.40.Kp, 73.63.Hs
\end{abstract}

The interplay between single particle localization and electron-electron interactions in disordered electronic systems has been under much investigation for two decades [1]. Due to disorder induced single particle localization, 2D non-interacting electron systems are predicted to be insulators at zero temperature in the presence of any disorder [1]. It was also widely accepted that adding electron interactions does not change this conclusion and, thus, there is no true metallic state in $2 \mathrm{D}$ at $T=0$. It came as a surprise when a $2 \mathrm{D}$ metallic state and metal-insulator transition(MIT) were observed in various high mobility low density 2D systems after the initial discovery of Kravchenko et al. 2]. The strong Coulomb interactions in these low density metallic systems revived interest in the role of Coulomb interactions in disordered $2 \mathrm{D}$ systems.

A comprehensive theoretical understanding of the Coulomb interaction effects on the 2D electron transport has emerged over the years 3, 4, 5, 6, 7]. For diffusive electrons at low $T$, Coulomb interactions are known to give a $\ln T$ conductivity correction $\delta \sigma(T)$, accompanying the similar $\ln T$ correction from single particle interference in the weakly disordered regime 3, 4]. Recently Zala, Narozhny and Aleiner(ZNA) pointed out that the logarithmic Altshuler-Aronov interaction correction to $\sigma$ originates from coherent scattering of Friedel oscillations. They extended the calculation to intermediate temperatures where transport is ballistic $\left(k_{B} T>\hbar / \tau\right)$ instead of diffusive $\left(k_{B} T<\hbar / \tau\right)$ []]. For high mobility samples exhibiting $2 \mathrm{D}$ metallic conduction, the elastic scattering time $\tau$ is large and the sample is usually in the ballistic regime. In this regime, ZNA showed that $\delta \sigma(T)$, the interaction correction, could be positive('metallic') or negative(insulating), depending on the FL parameter $F_{0}^{\sigma}$ just as in the diffusive regime. The ZNA theory improves the previous screening theory of Coulomb interactions at intermediate temperatures $[5,6 \mathrm{a}, \mathrm{b}]$, and predicts a linear $T$-dependent $\delta \sigma(T)$ controlled by $F_{0}^{\sigma}$.

The interaction correction theory of FL systems in the ballistic regime [7] was applied by various experimental groups to explain the zero magnetic field metallic conductivity 8, 9, 10, 11, 12, 13, 14]. In these analyses, negative $F_{0}^{\sigma}$ 's were obtained from fitting the metallic $\sigma(T)$ to a linear function of $T$ as predicted by the ZNA theory. In the FL theory, a negative(positive) $F_{0}^{\sigma}$ corresponds to ferromagnetic(antiferromagnetic) spin exchange interaction. While various scattering mechanisms besides the interaction correction can contribute to the longitudinal conductivity, the $T$ dependent Hall resistivity is a good probe for separating the Coulomb interaction effects [3, 15, 16, 17]. In this paper we present an analysis of the temperature dependent Hall resistivity together with the longitudinal conductivity of a metallic 2D hole system within the recent ballistic FL theory in both weak [7] and strong perpendicular magnetic field [18]. We found that for all the densities studied, the slope of $\rho_{x y}(B)$ is enhanced by a decreasing temperature and/or increasing magnetic field. When the $B=0$ metallic conductivity is used to fix the FL parameters, analysis shows that the enhanced slope of $\rho_{x y}(B)$ is qualitatively and quantitatively inconsistent with interaction corrections to Fermi liquid theory.

We performed the experiments on two dilute 2D hole systems in two $10 \mathrm{~nm}$ wide GaAs quantum wells. The samples were made from the same wafer used in our previous study [19]. The hole density $p$ was tuned by a gold backgate which is about $150 \mu \mathrm{m}$ underneath the quantum well. The two samples were measured in two different toploading Helium3-4 dilution refrigerators: sample A was mounted on the copper tail of the mixing cham- 
ber of the refrigerator at UC-Riverside, while sample B was immersed in the liquid Helium3-4 mixture inside the mixing chamber of the refrigerator at LANL. The data collected from the two samples in the two refrigerators are consistent with each other even down to our lowest experimental temperature of $20 \mathrm{mK}$. During the measurements, the voltage applied to the sample was always kept low (typically a few microvolts) such that the power delivered to the sample is less than a few fWatts $/ \mathrm{cm}^{2}$ to avoid overheating the holes.

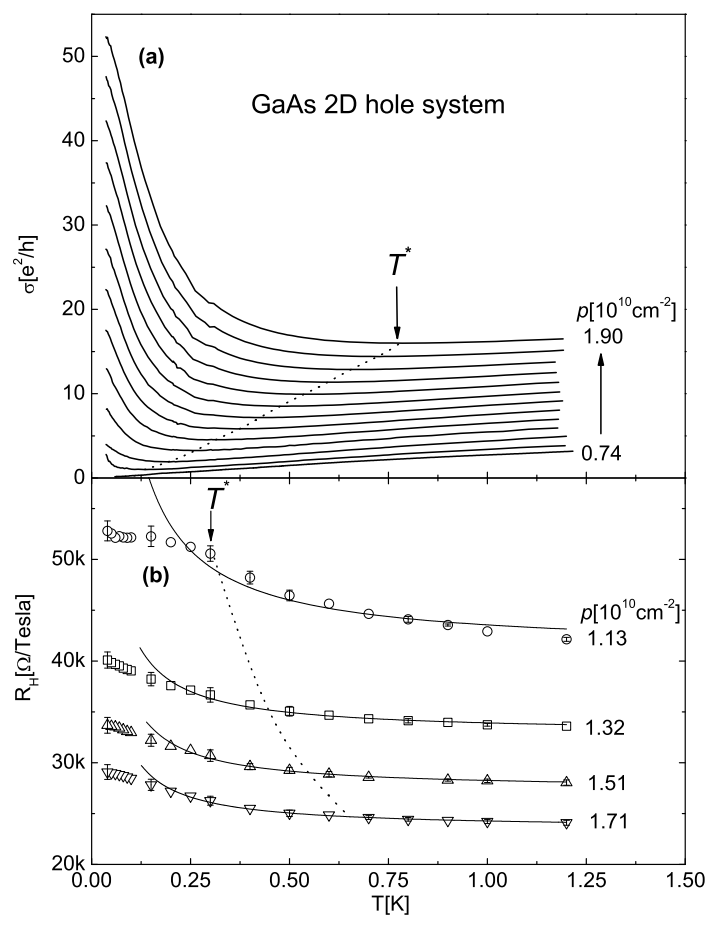

FIG. 1: (a)The $B=0$ temperature dependent conductivity $\sigma(T)$ of $2 \mathrm{D}$ holes with thirteen different densities in a $10 \mathrm{~nm}$ wide GaAs quantum well (sample A). The hole density spans from $p=0.74$ to $1.9 \times 10^{10} \mathrm{~cm}^{-2}$ with $0.965 \times 10^{9} \mathrm{~cm}^{-2}$ step from the bottom curve to the top curve. The MIT of this sample happens around $p_{c} \sim 0.78 \times 10^{10} \mathrm{~cm}^{-2}$. (b)The temperature dependent Hall coefficients for four densities in (a). The black lines depict the functional behavior const. $+1 / T$. For $p>p_{c}$, a dotted line is plotted in both panels to indicate the temperature $T^{*}$ where the sample turns metallic.

In Fig प, we present the temperature dependent conductivity $\sigma(T)$ of sample A for various hole densities $\left(p=0.74-1.9 \times 10^{10} \mathrm{~cm}^{-2}\right)$ at $B=0$. The density is determined from the Shubnikov-de Haas $(\mathrm{SdH})$ oscillations. For all the densities except $0.74 \times 10^{10} \mathrm{~cm}^{-2}, \sigma(T)$ turns from insulating-like $(\mathrm{d} \sigma(T) / \mathrm{d} T>0)$ to metalliclike $(\mathrm{d} \sigma(T) / \mathrm{d} T<0)$ below a characteristic temperature $T^{*}$. The metallic $\sigma(T)$ for $p>p_{c}$ below $T^{*}$ was recently attributed by some authors to the Coulomb interaction correction of a Fermi liquid with $F_{0}^{\sigma}<0$ at in- termediate temperatures according to the ZNA theory [8, 9, 10, 11, 12, 13, 14]. Theoretically, interaction effects will also give a correction to the Hall resistivity. In the low $T$ diffusive limit, interactions have a correction $\delta R_{H}(T) \sim \ln T$ to $R_{H}$, the Hall coefficient(the slope of $\rho_{x y}(B)$ in small $\left.B\right)[3]$. In the ballistic regime, $\delta R_{H}(T)$ is expected to change to a $1 / T$ dependence [7]. Thus, depending on the value of $F_{0}^{\sigma}, R_{H}$ will increase or decrease towards the Drude Hall coefficient as $R_{H}(T) \sim 1 / T$ when $T$ increases. Fig पp presents the $R_{H}$ vs. $T$ data for four metallic densities in Fig प. $R_{H}$ was obtained by linearly fitting $\rho_{x y}(B)$ between $-0.05 \mathrm{~T}$ and $+0.05 \mathrm{~T}$ perpendicular field. It can be seen that at temperatures above $0.1 \mathrm{~K}$ the measured $R_{H}(T)$ may be described as a const. $+1 / T$ function(Fig \), although the fit fails at lower temperatures where the theory should apply best.

Now we quantitatively discuss the longitudinal transport together with the Hall resistivity within the interaction correction theory of FL, using a density $\left(p=1.65 \times 10^{10} \mathrm{~cm}^{-2}\right)$ in sample B as an example. Fig 2 presents $\sigma(T)$ at $B=0$. In the ballistic regime, the interaction correction to conductivity is []

$$
\delta \sigma(T)=\sigma_{D}\left(1+\frac{3 F_{0}^{\sigma}}{1+F_{0}^{\sigma}}\right) \frac{T}{T_{F}} .
$$

Following the analyses of ref. [8, 9, 10, 11, 12, 13, 14], we also can fit the $B=0$ conductivity data for $0.1 \mathrm{~K}<$ $T<0.2 \mathrm{~K}$ to the linear dependence of Eq. 1 obtaining a Drude conductivity of $40 e^{2} / h$ and $F_{0}^{\sigma}=-0.6$. The hole mass was set to be $m^{*}=0.38 m_{e}$ in the fitting process, with $m_{e}$ being the free electron mass. In Fig 20, $R_{H}$ vs $T$ data are plotted together with the predicted $R_{H}(T)$ (the gray line) according to ZNA theory with $\sigma_{D}=40 e^{2} / h$ and $F_{0}^{\sigma}=-0.6$. In the ZNA theory, the interaction correction to $R_{H}$ is the summation of the corrections from the singlet(charge) channel and the triplet(spin) channel: $\delta R_{H}=\delta R_{H}^{\rho}+\delta R_{H}^{\sigma}$. The singlet channel correction $\delta R_{H}^{\rho}$ and the triplet channel correction $\delta R_{H}^{\sigma}$ are given as Eq.17, Eq.18 respectively in ref.5c.

The discrepancy between the data and theoretical expectation in the metallic regime of Fig. 2 is obvious. In fact, for $F_{0}^{\sigma}=-0.6$, the theory predicts a nearly flat but decreasing $R_{H}$ as temperature decreases in the experimental temperature range $(20 \mathrm{mK}-1.2 \mathrm{~K})$. Note that the FL theory predicts the interaction correction to $R_{H}$ to be very small in the ballistic regime for large $\sigma_{D}$, consistent with the Hall coefficient measurements for metallic 2D electrons in high mobility Silicon-metal-oxidesemiconductor field-effect transistors(Si-MOSFET's) 20, 21, 22.

It is important to know if the temperature enhanced $R_{H}$ is actually related to a varying carrier density effect. A standard way to measure carrier density is the $\mathrm{SdH}$ oscillations in the longitudinal magneto-resistivity $\rho_{x x}(B)$. From the positions of the $\mathrm{SdH}$ minima/maxima 


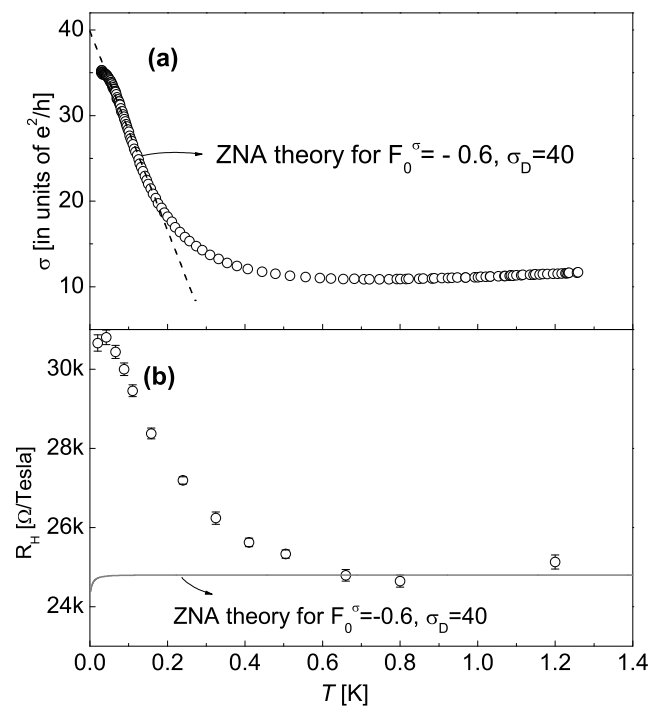

FIG. 2: (a) Conductivity $\sigma(T)$ for 2D holes with $p=1.65 \times 10^{10} \mathrm{~cm}^{-2}$ in sample B. The dashed black line is the linear fit of the $B=0$ metallic $\sigma(T)$ according to the FL interaction correction theory of ZNA, which yields $F_{0}^{\sigma}=-0.6$ and Drude conductivity $\sigma_{D}=40$. (b)Comparison of the $R_{H}(T)$ data for $2 \mathrm{D}$ holes in (a) with the theoretical expectation assuming the $B=0$ metallic conductivity is due to interaction correction of a FL. The gray line is the theoretical curve for $F_{0}^{\sigma}=-0.6$ and $\sigma_{D}=40$.

one can extract the carrier density. At $20 \mathrm{mK}$ we could observe $\mathrm{SdH}$ oscillation in $\rho_{x x}(B)$ down to $\sim 0.06 \mathrm{~T}$. Note that resolving $\mathrm{SdH}$ at low magnetic fields(high filling factors) is difficult for low density holes with large effective mass(and hence small cyclotron energy) because of the necessity to cool the holes to very low temperature. Fig 3 shows the index number vs. $1 / B$ for the positions of the $\mathrm{SdH}$ oscillations shown in the inset. We obtain the total hole density $p=1.74 \times 10^{10} \mathrm{~cm}^{-2}$ and the majority $/$ minority spin subband densities $p_{+/-}=1.15,0.59 \times$ $10^{10} \mathrm{~cm}^{-2}$, via linear fitting of the index number vs. $1 / B$ following ref. 23, 24. The analysis of $\mathrm{SdH}$ beating is consistent with a fixed ( $B$-independent) density(with $30 \%$ net spin polarization at $B=0)$ in the regime of $\mathrm{SdH}$ oscillations and quantum Hall plateaus 25. However, the low-field $(\leq 0.05 \mathrm{~T})$ slope of the Hall coefficient, $R_{H}(T)$, changes by more than $20 \%$ between 0.1 and $0.5 \mathrm{~K}$, temperatures sufficiently high that most $\mathrm{SdH}$ oscillations at high filling factors are no longer observable. Nevertheless, the positions of the $\mathrm{SdH}$ dips at $\nu=1,2$ do not move with $T$, and hence strongly imply a fixed ( $T$-independent) carrier density. The $T=20 \mathrm{mK} \mathrm{SdH}$ oscillations and Hall resistivity $\rho_{x y}(B)$ are presented in Fig $3 \mathbf{b}$. The data are averaged from both positive and negative magnetic field measurements to remove the admixture between $\rho_{x x}$ and $\rho_{x y}$. We
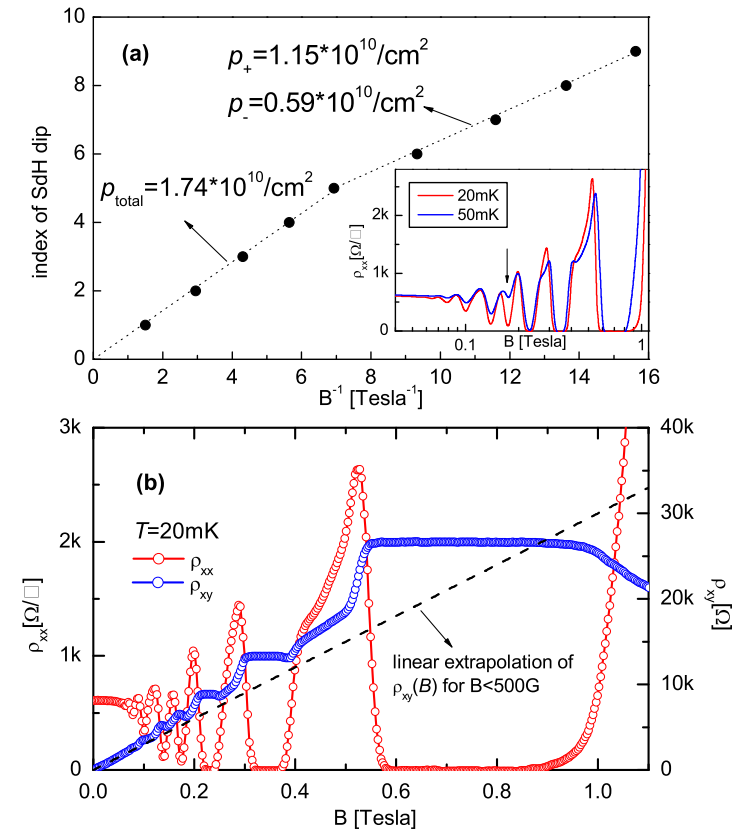

FIG. 3: (color online)(a)The index number $i$ vs. $1 / B$ of SdH dips for sample B in Fig.2. Linear fitting $i$ vs. $1 / B$ in high $B$ region yields a total hole density $p=1.74 \times 10^{10} \mathrm{~cm}^{-2}$. Linear fitting the low $B$ part gives the densities for majority/minority spin subband $p_{+/-}=1.15,0.59 \times 10^{10} \mathrm{~cm}^{-2}$. The inset shows the $\mathrm{SdH}$ oscillations at $20 \mathrm{mK}$ and $50 \mathrm{mK}$, with an arrow marking the beating node around $0.15 \mathrm{~T}$. (b)Longitudinal resistivity $\rho_{x x}$ and Hall resistivity $\rho_{x y}$ at $20 \mathrm{mK}$. The quantized Hall plateaus and $\mathrm{SdH}$ minima coincide, yielding a density that is $\sim 20 \%$ smaller than deduced from the linear extrapolation (dashed line) of $\rho_{x y}$ from low fields $(<0.05 \mathrm{~T})$.

see that the SdH dips and quantized Hall plateaus occur at the same magnetic fields. Note, however, that the extrapolation of the low $B(\leq 0.05 \mathrm{~T}) \rho_{x y}$ (dashed line) intersects the Hall plateaus at magnetic fields higher than the plateau centers, indicating that the low field $R_{H}$ is smaller than that determined at high fields. While this $20 \%$ discrepancy could, in principle, be due to interaction corrections to $R_{H}[3,15,16,17$, we have already shown that the $\sigma(T)$ and $R_{H}(T)$ data are not explained consistently within the interaction theory of FL.

While ZNA's theory is only applicable in the low field limit $\left(\omega_{c} \tau<1\right)$, Gornyi and Mirlin(GM) recently calculated the interaction correction to $\rho_{x y}$ into the high magnetic field regime $\left(\omega_{c} \tau \gg 1\right)$ with $\omega_{c}=e B / m^{*}$ being the cyclotron frequency [18]. We also investigated the behavior of $\rho_{x y}(B)$ in strong magnetic fields to further test the FL interaction correction theory for our sample. In GM's strong magnetic field theory, the interaction correction to $\rho_{x y}$ is separated into two parts. One part is $T$-dependent but $B$-independent, and the other part is 


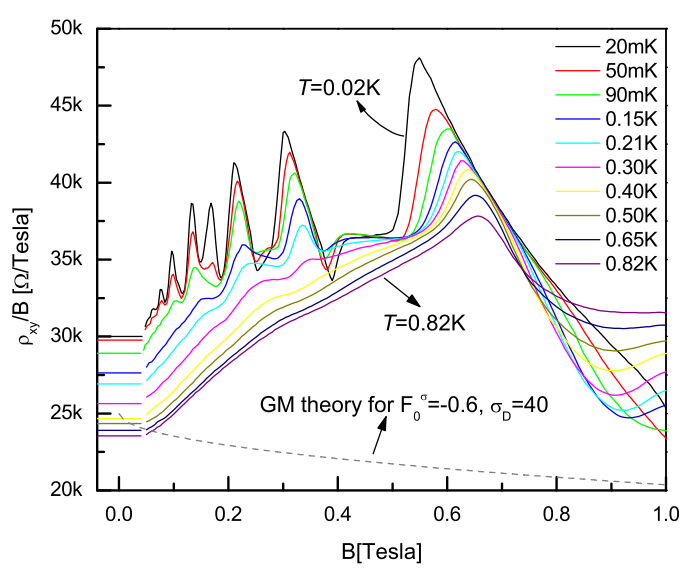

FIG. 4: (color online) $\rho_{x y} / B$, the slope of Hall resistivity vs. magnetic field. When $B$ is strong $\left(\omega_{c} \tau \gg 1\right)$, a $\sqrt{B}$ dependent correction to $\rho_{x y} / B$ is expected in the FL theory [18]. The dashed line is the theoretical curve for parameters $F_{0}^{\sigma}=-0.6$ and $\sigma_{D}=40$ and a zero field value of $\rho_{x y} / B=25 \mathrm{k} \Omega / \mathrm{T}$.

$B$-dependent and $T$-independent. In Fig 4 we plot the Hall slope, $\rho_{x y} / B$ vs. $B$ at various temperatures. To remove the admixture of $\rho_{x x}$ into $\rho_{x y}$, we antisymmetrized the $\rho_{x y}$ data from both $B>0$ and $B<0$ measurements to obtain Fig 4 . The low field $(B \leq 500 \mathrm{G}) R_{H}$ data are also included. Fig 0 shows that the $\rho_{x y} / B$ data indeed may be viewed as a $T$-independent magnetic field enhancement on the background of a $B$-independent temperature enhancement 26]. The interaction correction to $\rho_{x y}$ at strong $B$ is also quantitatively related to the FL parameter $F_{0}^{\sigma}$ as in ZNA theory [18]. The $T$-dependent part of the $\rho_{x y}$ correction in the ballistic regime and strong $B$ is 18

$$
\frac{\delta \rho_{x y}^{T}}{\rho_{x y}}=-7.117 \frac{e^{2} / \hbar}{\sigma_{D}}\left(\frac{3 F_{0}^{\sigma}}{F_{0}^{\sigma}+1}+1\right)\left(\frac{k_{B} T}{\hbar / \tau}\right)^{1 / 2} .
$$

In this high field regime, as in the low field regime, theory predicts a decreasing slope of the Hall resistivity with decreasing temperature. However, the opposite behaviour, i.e. enhancement of the Hall resistivity, is observed when $T$ decreases. The $B$-dependent part of the GM correction to $\rho_{x y}$ is 18

$$
\frac{\delta \rho_{x y}^{B}}{\rho_{x y}} \approx \frac{e^{2} / \hbar}{\sigma_{D}}\left(\frac{3 F_{0}^{\sigma}}{F_{0}^{\sigma}+1}+1\right)\left(\omega_{c} \tau\right)^{1 / 2} .
$$

Fig 4 also includes the theoretical curve from $\mathrm{Eq}[3$ for $F_{0}^{\sigma}=-0.6, \sigma_{D}=40$ and $\rho_{x y} / B(B=0)=25 \mathrm{k} \Omega / \mathrm{T}$. One can see that $\delta \rho_{x y}^{B} / \rho_{x y}$ is expected to be negative for $F_{0}^{\sigma}=-$ 0.6 but the data show a positive increase as $B$ increases.

Fig 4 also suggests that $\rho_{x y} / B$ is enhanced with decreasing $T$ at both weak and strong magnetic fields in a similar fashion. It is reasonable to conclude that the $T$ dependent $\rho_{x y} / B$ originates from the same mechanism for both magnetic field regimes. Since our temperature dependent SdH shows that the enhanced $\rho_{x y} / B$ at high $B$ is not related to a temperature dependent density, we further conclude that the enhanced low magnetic field Hall coefficient is not due to a density effect. In conclusion, for both the low magnetic field(ZNA) and high magnetic field(GM) regimes our combined resistivity and Hall data are inconsistent with the electron interaction corrections interpretation in a Fermi liquid.

Finally, we briefly comment on the relevance between our data and several other FL-based models of the 2D metallic state, which do not invoke the FL parameters $[6,27]$. For our sample in the metallic state, $\sigma$ is enhanced as large as three times as $T$ is reduced, a result perhaps consistent with the screening theory of Das Sarma and Hwang [6]; however, the behavior of $R_{H}$ has not yet been theoretically discussed within the screening theory. Alternatively, the enhanced $R_{H}$ at low $T$ could be interpreted as a carrier freeze out[ref.6a] or trapping effect 27]; however, the field $(B>0.06 \mathrm{~T})$ and temperature independent density we observe in the $\mathrm{SdH}$ oscillations require these effects to disappear above $0.06 \mathrm{~T}$ and make these interpretations seem highly unlikely.

The authors are pleased to thank I.L. Aleiner and A. Punnoose for valuable discussions. Work at UCR was supported by LANL-CARE program. The NHMFL is supported by the NSF and the State of Florida.

* Electronic address: xuangao@lanl.gov

[1] P.A. Lee and T.V. Ramakrishnan, Rev. Mod. Phys. 57, 287 (1985).

[2] E. Abrahams, S.V. Kravchenko, and M.P. Sarachik, Rev. Mod. Phys. 73, 251 (2001).

[3] B.L. Altshuler and A.G. Aronov, in Electron-Electron Interactions in Disordered Systems, edited by A.L. Efros and M. Pollak (North-Holland, Amsterdam, 1985).

[4] A.M. Finkelstein, Sov. Phys. JETP 57, 97 (1983).

[5] A. Gold and V. T. Dolgopolov, Phys. Rev. B 33, 1076 (1986).

[6] S. Das Sarma and E. H. Hwang, (a) Phys. Rev. Lett. 83, 164 (1999); (b)Phys. Rev. B 61, R7838 (2000);(c) ibid, 69, 195305 (2004).

[7] G. Zala, B.N. Narozhny, and I.L. Aleiner,(a) Phys. Rev. $B$ 64, 214204 (2001);(b) 65, 020201(R) (2002); (c)64, 201201 (2001).

[8] Y.Y. Proskuryakov et al., Phys. Rev. Lett. 89, 076406 (2002).

[9] P.T. Coleridge, A.S. Sachrajda, and P. Zawadzki, Phys. Rev. B 65, 125328 (2002).

[10] Z.D. Kvon,O. Estibals ,G.M. Gusev , and J.C. Portal, Phys. Rev. B 65, R161304 (2002).

[11] A.A. Shashkin,S.V. Kravchenko,V.T. Dolgopolov and T.M. Klapwijk, Phys. Rev. B 66, 073303 (2002).

[12] H. Noh et al., Phys. Rev. B 68, 165308 (2003). 
[13] V. M. Pudalov et al., Phys. Rev. Lett. 91, 126403 (2003).

[14] S. A. Vitkalov, K. James, B. N. Narozhny, M. P. Sarachik, and T. M. Klapwijk,Phys. Rev. B 67, 113310 (2003).

[15] D.J. Bishop, D.C. Tsui and R.C. Dynes, Phys. Rev. Lett. 46, 360 (1981).

[16] M.J. Uren, R.A. Davies and M. Pepper, J. Phys. C:Solid St. Phys. 13, L985 (1980).

[17] C.J. Emeleus et al., Phys. Rev. B 47, 10016 (1993).

[18] I.V. Gornyi and A.D. Mirlin, (a)Phys. Rev. Lett. 90, 076801 (2003); (b)Phys. Rev. B 69, 045313 (2004).

[19] X. P.A. Gao, A.P. Mills, Jr., A.P. Ramirez, L.N. Pfeiffer, and K.W. West, Phys. Rev. Lett. 89, 016801 (2002).

[20] V.M. Pudalov, G. Brunthaler, A. Prinz, and G. Bauer JETP Letters 70, 48 (1999).

[21] M.P. Sarachik, D. Simonian, K.M. Mertes, S.V. Kravchenko, and T.M. Klapwijk,Phyica B 280, 301 (2000).

[22] M. Khodas and A.M. Finkel'stein Phys. Rev. B 68, 155114 (2003).

[23] H.L. Stormer et al., Phys. Rev. Lett. 51, 126 (1983).
[24] J. P. Eisenstein, H. L. Stormer, V. Narayanamurti, A. C. Gossard, and W. Wiegmann Phys. Rev. Lett. 53, 2579 (1984).

[25] We found that the $B=0$ spin splitting increases from $20 \%$ to $32 \%$ as the density decreases from 2.35 to $1.35 \times 10^{10} \mathrm{~cm}^{-2}$ for sample B. Note that the inversion asymmetry related Rashba spin splitting should be negligible for our symmetrically doped quantum well with low hole density 24]. The $B=0$ spin splitting here is perhaps related to the strong ferromagnetic spin exchange interactions and ferromagnetic instability of 2D MIT in high $r_{s}$ 2D systems (A. A. Shashkin, S. V. Kravchenko, V. T. Dolgopolov, and T. M. Klapwijk Phys. Rev. Lett. 87, $086801(2001))$.

[26] The oscillatory behavior of $\rho_{x y} / B$ in Fig 4 at low temperature comes from the onset of quantum Hall effects.

[27] B. L. Altshuler and D. L. Maslov, Phys. Rev. Lett. 82, 145 (1999). 